\title{
Proper motions and CCD-photometry of stars in the region of the open cluster Trumpler $2^{\star}$
}

\author{
V. N. Frolov ${ }^{1}$, J. K. Ananjevskaja ${ }^{1}$, E. G. Jilinski ${ }^{1,2}$, D. L. Gorshanov ${ }^{1}$, and N. M. Bronnikova ${ }^{1, \star \star}$ \\ 1 The Main Astronomical Observatory, Pulkovo, St. Petersburg, Russia \\ e-mail:vfrol@gao.spb.ru \\ 2 Observatório Nacional / MCT, Rio de Janeiro, RJ, Brazil
}

Received 17 December 2004 / Accepted 29 December 2005

ABSTRACT

\begin{abstract}
The results of a complex study of the galactic open cluster Trumpler 2 are presented. In order to obtain the proper motions, the positions of approximately 3000 stars up to the limit magnitude $B \sim 16.25 \mathrm{mag}$ in the area $80^{\prime} \times 80^{\prime}$ around the cluster were measured on 6 plates with a maximal epoch difference of 63 years. The root-mean error of the relative proper motions is $4.2 \mathrm{mas} \mathrm{yr}^{-1}$. The catalogue of $B V$ magnitudes of all the stars in the investigated area was compiled. The selection of the cluster members within the region of $R<16^{\prime}$ from the center of the cluster was made. Altogether, 148 stars were considered to be cluster members by both astrometrical and photometrical criteria. We present the estimated age $\left(8.91 \times 10^{7} \mathrm{yr}\right)$ and physical parameters of the cluster and append the catalogues of the proper motions and of the photometry of the stars.
\end{abstract}

Key words. open clusters and associations: individual: Trumpler 2

\section{Introduction}

The open cluster Trumpler 2 is located near the famous rich clusters $h$ and $\chi$ in the Perseus constellation. Its equatorial and galactic coordinates are: $\alpha=2^{\mathrm{h}} 37^{\mathrm{m}} 15^{\mathrm{s}}, \delta=+55^{\circ} 59^{\prime} ; \ell=137^{\circ} .4$ $b=-3.89$ (2000.0). In the Trümpler (1930) catalogue it is classified as II $2 \mathrm{p}$ (a pure cluster with little central concentration). All that was known about the cluster to the present day is based on the data from the Washington catalogue "Photometry of stars in Galactic cluster fields" (Hoag et al. 1961, HJ). For Trumpler 2 it included the UBV photoelectrical photometry of 28 stars in the $15^{\prime} \times 15^{\prime}$ area and photographic photometry of 133 stars in the circle $R<22^{\prime}$ around the supposed cluster center. All the contemporary determinations of the cluster parameter are based on that data (Figs. 1a, b present the color-magnitude diagram (CMD) and the two-color diagram of the cluster from that study). The existence of the cluster itself was not confirmed by any other original papers. This area has never been investigated kinematically on the basis of proper motions. Our paper tries to fill this gap.

\section{Astrometry}

\subsection{Proper motions}

The observational material belongs to the Normal astrograph collection of the Pulkovo observatory and dates from 1937 to 2000 (Table 1). The scale of the Normal astrograph plates is $60^{\prime \prime} \mathrm{mm}^{-1}$. The cluster-region star positions from the USNO-A 2.0 catalogue were used as an additional plate (epoch 1954.8).

\footnotetext{
* Tables 2, 3A, 3B and 5 are only available in electronic form at the CDS via anonymous ftp to

cdsarc.u-strasbg.fr $(130.79 .128 .5)$ or via

http://cdsweb.u-strasbg.fr/cgi-bin/qcat?J/A+A/451/901

$\star \star$ Deceased.
}

All of the plates were scanned by means of the automated measuring complex "Fantasy" of the Pulkovo observatory. The description of the complex was presented in Frolov et al. (2002, FJA). The positions of the 2362 stars in the area $80^{\prime} \times 80^{\prime}$ centered on the cluster Trumpler 2 were measured up to the limit magnitude $B=16.25 \mathrm{mag}$. Positions of some faint stars were not measured on all plates because of the poor quality of their images, so from three to seven stellar positions were used to derive proper motions (PM), which were determined by the linemethod. The details of this method were presented in Jilinski et al. (2000). The deepest plate $K 376$ with the faintest images was considered to be the central plate. It was orientated by means of the stars' positions from the "Tycho-2" catalogue. Then all the other plates were reduced to this central one.

For that reduction 72 reference stars were selected in the interval between 13.5 and $14.5 \mathrm{mag}$. The rms errors of derived PM are: $\sigma_{x}= \pm 4.1 \operatorname{mas~yr}^{-1}, \sigma_{y}= \pm 4.2$ mas yr $^{-1}$. The analysis of error distributions demonstrates that they increased

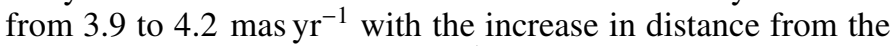
area center and from $3.1 \mathrm{mas}^{-1}$ for stars with the magnitude $B<10.5 \mathrm{mag}$ to 4.4 for $B>16 \mathrm{mag}$ stars. The astrometrical results are given in Table 2 with columns containing: 1 - the star number; 2 - the star number from the USNO-A 2.0 catalogue; $3,4-$ its rectangular coordinates $\mathrm{X}$ and $\mathrm{Y}$ on the plate $\mathrm{K} 376$; $5-$ radius-vector; $6-9-\mathrm{X}$ and $\mathrm{Y}$ relative proper motions and their errors; 10 - the number of used plates; 11 - photographical $B$-magnitude, resulting from the "Fantasy" measurements; 12 - notes (bl marks blends, bad - bad measurement, abs - absence of the star on the plates, BD and $A G$ - star identification).

\subsection{Members segregation}

The selection of cluster members was made for stars located in the circle with $R<16^{\prime}$ around the cluster center. That region 

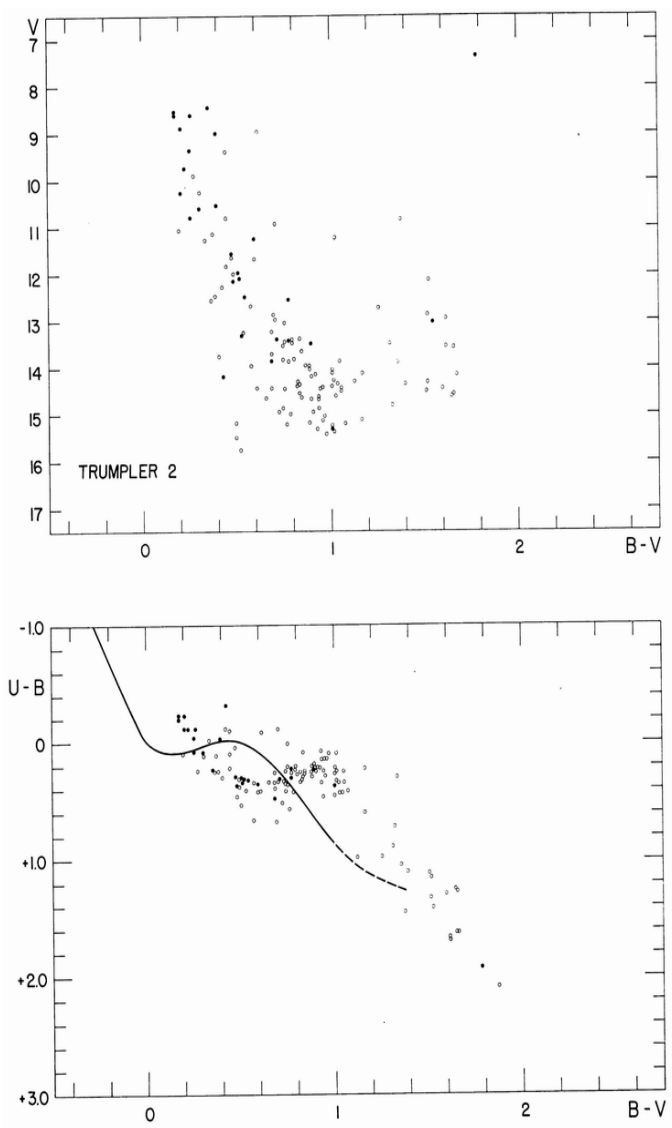

Fig. 1. Color-magnitude and two-color diagrams of Trumpler 2 from the Washington catalogue.

Table 1. Astrometric plates.

\begin{tabular}{lccl}
\hline \hline Plate & $\begin{array}{c}\text { Exposure } \\
\text { (min) }\end{array}$ & Epoch & Quality \\
\hline \multicolumn{4}{c}{ Early epoch } \\
K 376 & 25 & 1937 Sep. 12 & high \\
D 80 & 30 & 1949 Mar. 31 & good \\
D 135 & 30 & 1949 May 5 & poor \\
\multicolumn{4}{c}{ Recent epoch } \\
18160 & 25 & 1999 Dec. 12 & poor \\
18171 & 30 & 2000 Feb. 4 & good \\
18175 & 25 & 2000 Feb. 6 & good \\
\hline
\end{tabular}

contains 313 stars with determined proper motions. Blended images and stars measured only on two or less plates were expelled. The size of this circle was selected according to two criteria: first, it should be large enough not to miss the cluster members from the corona; second, not be too large to litter the vector point diagram (VPD) with a large amount of the field stars.

We used the method in which membership probabilities, magnitude equations (ME), and parameters of the star distributions on the vector point diagram (VPD) were determined step by step in an iterative process. The details of this procedure are described in FJA. The starting selection was realized in the circle with $R<10^{\prime}$ centered on the Trumpler2. As a first approximation, stars with the photoelectric $B V$ magnitudes from the HJ were considered to be the cluster members. Based on the proper motions and magnitudes of these stars the magnitude equation (ME) was studied and the proper motions of all stars were corrected for it. Then at each step that was characterized by

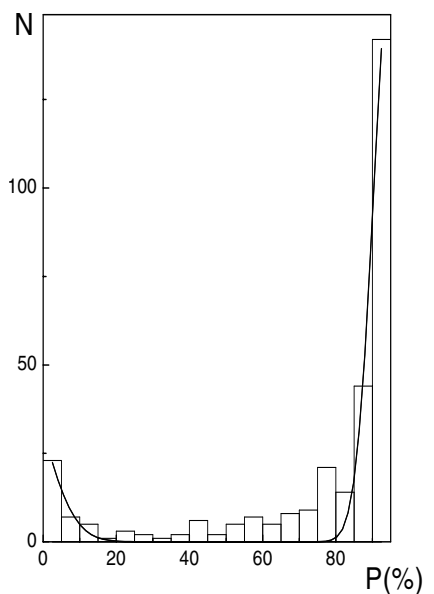

Fig. 2. Final histogram of star membership probabilities.

increasing value of $R\left(+2^{\prime}\right)$, the parameters of the bivariate Gaussian distributions of proper motions, circular for the cluster and elliptical for the field, were computed by the Sanders method (1971). The preliminary selection of the cluster members was made in each iteration step. Then the proper motions were tested for the ME. If it turned up, then all the PM in the catalogue were refreshed. The last step was made for the stars in the circle with radius $R<16^{\prime}$.

It is important to note that it is impossible to visually divide stars of the cluster and of the field on the VPD because of the proximity of the centers of their distributions. This fact is frequently registered for the far clusters (for example see Sanner et al. 1999, 2000, 2001). Nevertheless it takes only 8 iterations to reach the stable solution numerically with relative precision: $E=0.001$. The following initial values of the parameters were accepted: $N_{\mathrm{c}} / N=0.5$, centers of the distributions: the field $-1,1$, the cluster: $1,-1$, the dispersion: the field 12,8 , the cluster 4 . Calculations resulted in the following values: the relative number of cluster members and all stars $N_{\mathrm{c}} / N=0.74$; centers of distributions: the field $\mu_{x_{\mathrm{f}}}=0.98, \mu_{y_{\mathrm{f}}}=-2.11$; the cluster $\mu_{x_{\mathrm{c}}}=0.33, \mu_{y_{\mathrm{c}}}=-0.53$; standard deviations: the field $\Sigma_{x}=14.62, \Sigma_{y}=9.28$, the cluster $\sigma_{\mathrm{c}}=4.78$. All the values are in mas $\mathrm{yr}^{-1}$. They were used to calculate the individual membership probabilities of the stars in the area by the formulae:

$$
P\left(\mu_{x}, \mu_{y}\right)=\frac{N_{\mathrm{c}} \Phi_{\mathrm{c}}\left(\mu_{x}, \mu_{y}\right)}{N_{\mathrm{c}} \Phi_{\mathrm{c}}\left(\mu_{x}, \mu_{y}\right)+N_{\mathrm{f}} \Phi_{\mathrm{f}}\left(\mu_{x}, \mu_{y}\right)},
$$

where $N_{\mathrm{c}}$ is the normalized number of cluster stars, $N_{\mathrm{f}}$ the normalized number of field stars, $\mu_{x_{i}}$, and $\mu_{y_{i}}$ the proper motion in $x$ and $y$ for the $i$ th star.

The resulting histogram shows that the stars with the probabilities $P>85 \%$ can be considered as cluster members (Fig. 2). The whole amount of such stars is 192, and the final VPD is presented in Fig. 3a. Cluster members are plotted by the solid dots, and field stars by the crosses.

\section{Photometry}

The final conclusion about star membership was based on two criteria: its proper motion and its position on the CMD. Only a small part situated in the central circle stars has got the initial $U B V$-photometry (HJ). The $B$ and $V$ magnitudes of the rest of the stars were determined partly as a by-product of astrometric 


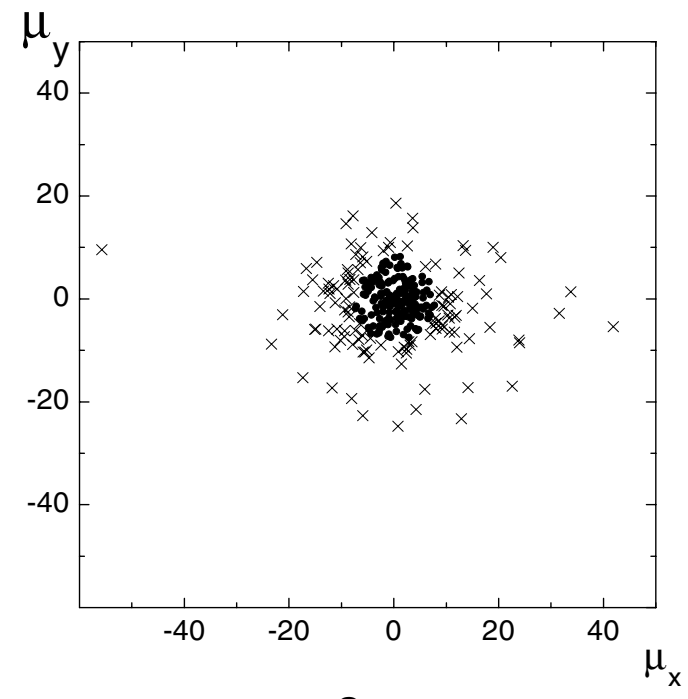

a

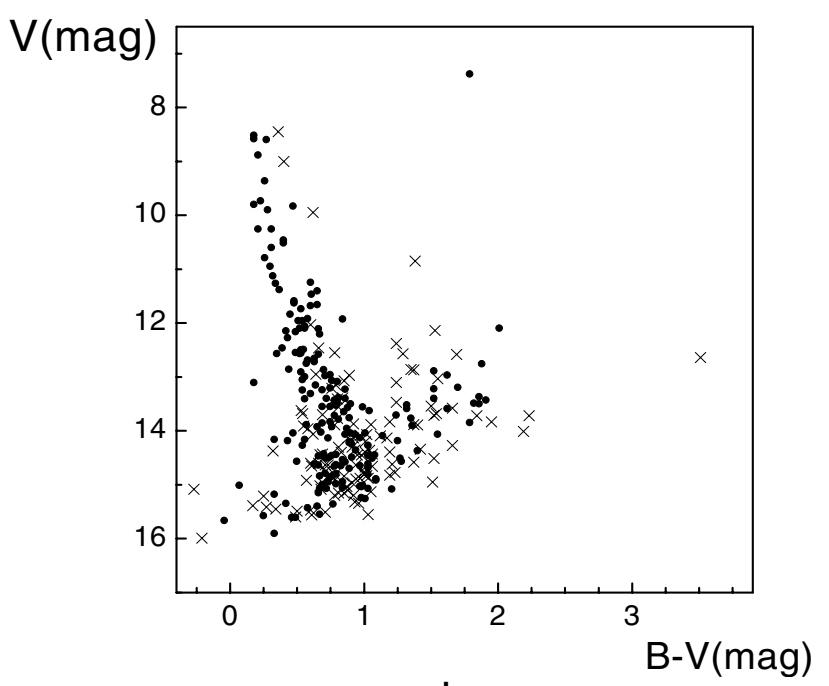

b

Fig. 3. a) VPD and b) CMD of the central part $\left(R<16^{\prime}\right)$ of Trumpler 2 . Astrometrical cluster members are plotted by solid dots, field stars by crosses.

plate measurements (image diameters) or by our original CCDobservations and were partly calculated from the infrared $J, H$, $K$ magnitudes from the 2MASS catalogue by the derived transformation formulae. Unfortunately, for technical reasons, we were only able to obtain $C C D$-magnitudes in the area of $17^{\prime} \times 24^{\prime}$. The identification map of the central part of the cluster is presented in Fig. 4. The star number from the Washington catalogue is indicated in front of the authors' number. Data available from the all the different sources of the broadband photometry for each star are presented in Table 3A, where columns contain: 1 - the star number according to Table 2;2 - the radius-vector; 3 - the specific numbers from 1 to 10 corresponding to photometry data; $3,4-B$ and $V$ magnitude; $5-B-V ; 6$ - notes. The description and corresponding comments of the specific numbers that mark the sources of photometry are given in in Table 4, whose columns contain: 1 - the specific number $(S / N) ; 2-$ photometry band; 3 - source of the material; 4 - the area dimensions in arcmin; 5 - the photometric errors in magnitudes.

Notes for specific numbers:

1,2. While plotting the characteristic curve $V$, the systematic difference between the photoelectric and photographic values $\left(V_{\mathrm{pe}}-V_{\mathrm{pg}}\right)=0$. 01 in the $\mathrm{HJ}$ catalogue was detected, and was taken into account. The same difference was detected for the colors $B-V$.

3. The real precision of the photometric data given in the Tycho-2 catalogue for stars $V_{\mathrm{T}}>9$ mag is much lower then claimed. That's why we preferred alternative sources for these stars.

4-6. B-photographic values of the majority of the stars in the studied region were determined by measuring their diameters by means of the "Fantazy" complex. It is well known that $B$ magnitudes obtained with the Pulkovo Normal astrograph are infected by the color equation (CE), which is why characteristic curves $B \sim F(D)$ were plotted separately for "red" $(B-V \geq 1.0)$ and "blue" $(B-V<1.0)$ stars. The $B$ magnitudes of the "blue" stars were not corrected for $\mathrm{CE}$ as its value was non-significant (smaller than the mean error). At the same time as the original observations made of the "red" stars, the value of the correction was 0.35 mag per magnitude and was taken into account in all cases.
4. $\quad V$-magnitudes were obtained from original the observations made with the 320-mm mirror astrograph ZA-320 of the Pulkovo Observatory equipped with the CCD-receiver ST-6 with a fixed TC241 matrix (Bekyashev et al. 1998).

5. $V$-magnitudes were calculated with the $J, H, K$ magnitudes from the infrared 2MASS catalogue by the derived transformation formula:

$$
\begin{aligned}
& V=0.02528+1.04259 J-0.83847(J-H) \\
& +3.16435(J-K)+2.39154(J-H)^{2}-1.34984(J-K)^{2} .
\end{aligned}
$$

7. $B$-magnitudes from the USNO-2A catalogue were treated only as control values.

8. $\quad B, V$ magnitudes were obtained from original observations with the $320 \mathrm{~mm}$ mirror astrograph ZA-320 of the Pulkovo Observatory equipped with the CCD-receiver ST-6 with a fixed TC241 matrix (Bekyashev et al. 1998).

9, 10. $B$-magnitudes were calculated with the $J, H, K$ magnitudes from the infrared 2MASS catalogue by the derived transformation formulae:

$$
\begin{aligned}
& B=1.0621 J+1.0732(J-H)+2.9973(J-K) \\
& \quad+0.3785(J-H)^{2}+0.4259(J-K)^{2} \\
& B-V=-0.21236+0.04164 J-2.51276(J-H) \\
& +2.43958(J-K)+2.27583(J-H)^{2}-0.14797(J-K)^{2} .
\end{aligned}
$$

Table 3B contains the additional magnitudes $B$ and $V$ obtained by means of the CCD-photometry for stars whose brightnesses are less than the limit sensitivity of the astrometric plates. Their numbers begin with the 3 on the identification map of the central part of the cluster (Fig. 4). Columns in Table 3B contain: 1 - the star number; $2-4-B$-magnitude, its error and the number of observations; 5-7 $-V$-magnitude, its error and the number of observations. As the standards for these observations we took the Washington photoelectric and partly (for the faint stars) photographic photometry. Errors of the magnitude determination were defined as external relative to the standards. Photometric data from Table 3A were used to construct the CMD for 313 stars in the circle $R<16^{\prime}$. As was mentioned in the process of membership determination, the positions of stars on the CMD were used as a second parameter. Because of the unequal accuracy of the material, the following application priority order of the specific 


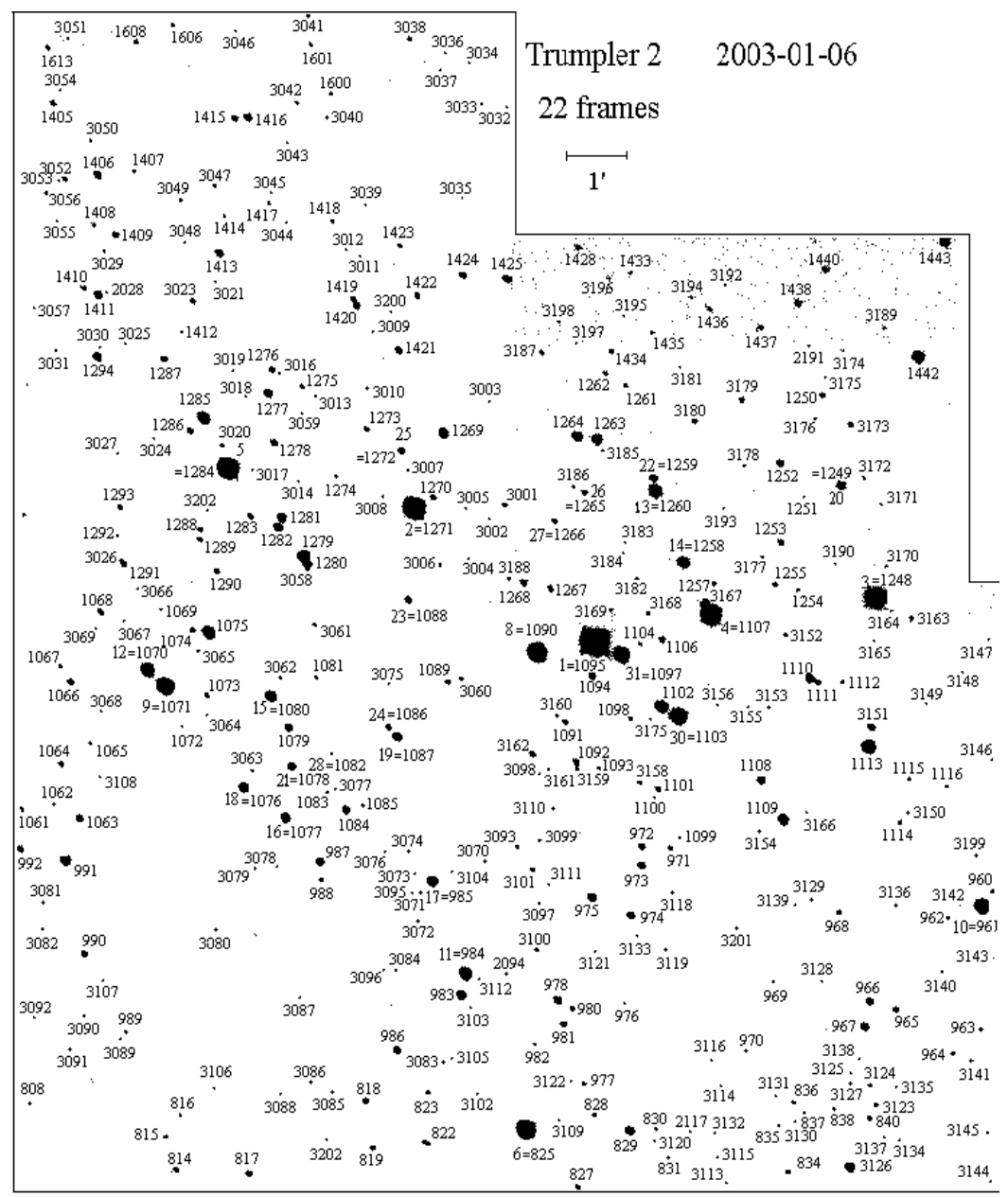

Fig. 4. CCD map of the central part of the cluster Trumpler 2. The numbers of stars are given according to the photometric study.

numbers $(S / N)$ was set: 1 (photoelectric $H J)-28$ stars; 2(photographic HJ) - 56 stars; 3 (Ticho) - 6 stars, 8 (CCD) - 95 stars, 4(the combination of authors' photographic and CCD) - 1 star, 5 - (the combination of authors' photographic and 2MASS) 127 stars. The CMD of all these stars is shown in Fig. $3 b$.

\section{Analysis and conclusion}

Stars of the IV and V luminosity classes falling on the CMD into the $\pm 3 \sigma_{B-V}$ interval from the cluster main sequence are considered to be the photometric cluster members. As the average error of the color index determination rises from the bright to faint stars, the width of the MS band increases approximately from $0.02 \mathrm{mag}$ to $0.11 \mathrm{mag}$.

In order to determine the mean absorption in the region of the cluster, the two-color diagram $(U-B) \sim(B-V)$ was plotted using the HJ $U B V$ photometry only for the 44 astrometric cluster members with $P \geq 85 \%$ falling on the MS. For the inclination $E(U-B) / E(B-V)=0.72$, the best coincidence of the line of normal colors with the two-color sequence of the cluster was achieved at the value of the color excess $E(B-V)=0.40 \pm 0.06$ mag (Fig. 5) that differs from what is accepted in existing compiled catalogues values: 0.30 (Hoag 1966) and 0.34 (Electronic Database WEBDA, 2001 ${ }^{1}$ ). The superposition of the MS of the cluster with the ZAMS (Schmidt-Kaler 1965) was made with this fixed value of color excess. During

${ }^{1}$ http://obswww. unige.ch/webda this procedure, special attention was paid to the stars with photoelectric photometry. The best coincidence was achieved at the value of the apparent distance module $\left(V-M_{V}\right)=10.50 \pm 0.35$, which corresponds to the true module $\left(V-M_{V}\right)_{0}=9.30$ and the distance $r=725 \mathrm{pc}(610<r<851)$. The CMD diagram for probable cluster members (according to both photometric and astrometric criteria) is presented in Fig. 6. Solid circles mark the stars supplied by photoelectric photometric data, and hollow circles all the others.

The catalogue of the stars in the central circle $\left(R<16^{\prime}\right)$ is presented in Table 5. Its columns contain $1-$ the author's number; 2 - sign of membership; 3, 4 - the rectangular coordinates of star $(\operatorname{arcmin}) ; 5$ - the distance from the center $(\operatorname{arcmin})$; 6-9 - the proper motions and their root-mean-square errors $\left(\right.$ mas $\left.\mathrm{yr}^{-1}\right) ; 10$ - the number of the astrometric plates; 11 - the membership probability; 12 - the photometry specific author's number (see Table 4); 13, 14 - the star magnitude and color.

Located in the cluster's center, the red giant (st. N 1095) has high individual membership probability. Large dispersion in its photometric data may be evidence of its variability. Although it does not fall in the $16^{\prime}$ circle where the individual membership probabilities were determined, another red giant (st. N 1748) relates to the third class of Eibighausen (1942) classification. This means that this star lies within the circle of the $R=2 \sqrt{2} \sigma$ on the VPD where $98 \%$ of all cluster members are situated (Kadla 1966). The same refers to st. N 1941, which must have travelled far from the cluster center but is gravitationally linked to it. 
Table 4. Cross-references for the photometry.

\begin{tabular}{|c|c|c|c|c|}
\hline 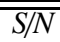 & 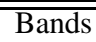 & Sources & Area & Errors \\
\hline 1 & $B, V$ & HJ, photoelectric magnitudes & $17^{\prime} \times 17^{\prime}$ & $\sigma_{V}= \pm 0.021 \mathrm{mag}, \sigma_{B-V}= \pm 0.018 \mathrm{mag}$ \\
\hline 2 & $B, V$ & HJ, photographic magnitudes & $30^{\prime} \times 12^{\prime}$ & $\sigma_{V}= \pm 0.041 \mathrm{mag}, \sigma_{B-V}= \pm 0.050 \mathrm{mag}$ \\
\hline 3 & $B_{\mathrm{T}}, V_{\mathrm{T}}$ & Tycho catalogue & & $\sigma_{V}= \pm 0.060 \mathrm{mag}$ \\
\hline & & & & $\begin{array}{l}\sigma_{B-V}= \pm 0.100 \mathrm{mag}(\text { all stars }) \\
\sigma_{V}= \pm 0.012 \mathrm{mag}\end{array}$ \\
\hline \multirow{3}{*}{4} & & & & $\sigma_{B-V}= \pm 0.019 \mathrm{mag}\left(V_{\mathrm{T}}<9 \mathrm{mag}\right)$ \\
\hline & $B$ & $\begin{array}{l}\text { Author's photographic magnitudes } \\
\text { catalogue }\end{array}$ & $120^{\prime} \times 120^{\prime}$ & $\begin{array}{l}\sigma_{B}= \pm 0.09 \mathrm{mag} \text { for "red" stars } \\
\sigma_{B}= \pm 0.03 \mathrm{mag} \text { for "blue" stars }\end{array}$ \\
\hline & $V$ & $\begin{array}{c}\text { Author's } C C D \text { magnitudes } \\
\text { catalogue }\end{array}$ & $19^{\prime} \times 24^{\prime}$ & $\sigma_{V}= \pm 0.06 \mathrm{mag}$ \\
\hline \multirow[t]{3}{*}{5} & $B$ & $\begin{array}{l}\text { Author's photographic magnitudes } \\
\text { catalogue }\end{array}$ & $120^{\prime} \times 120^{\prime}$ & $\begin{array}{l}\sigma_{B}= \pm 0.09 \text { mag for "red" stars } \\
\sigma_{B}= \pm 0.03 \text { mag for "blue" stars }\end{array}$ \\
\hline & $V$ & 2MASS catalogue & & $\sigma_{V}= \pm 0.11 \mathrm{mag}$ \\
\hline & & calculated from the $J, H, K$ magnitudes & & \\
\hline \multirow[t]{2}{*}{6} & $B$ & $\begin{array}{l}\text { Author's photographic magnitudes } \\
\text { catalogue }\end{array}$ & $120^{\prime} \times 120^{\prime}$ & $\begin{array}{l}\sigma_{B}= \pm 0.09 \mathrm{mag} \text { for "red" stars } \\
\sigma_{B}= \pm 0.03 \mathrm{mag} \text { for "blue" stars }\end{array}$ \\
\hline & $V$ & No data & & \\
\hline \multirow[t]{2}{*}{7} & $B$ & USNO $2 \mathrm{~A}$ catalogue & & \\
\hline & $V$ & No data & & \\
\hline \multirow[t]{2}{*}{8} & $B$ & $\begin{array}{c}\text { Author's } C C D \text { magnitudes } \\
\text { catalogue }\end{array}$ & $19^{\prime} \times 24^{\prime}$ & $\sigma_{B}=0.08 \mathrm{mag}$ \\
\hline & $V$ & $\begin{array}{c}\text { Author's } C C D \text { magnitudes } \\
\text { catalogue }\end{array}$ & $19^{\prime} \times 24^{\prime}$ & $\sigma_{V}=0.05 \mathrm{mag}$ \\
\hline \multirow[t]{2}{*}{9} & $B$ & $\begin{array}{l}\text { calculated from the values of } B-V \\
\text { derived from the } 2 \text { MASS catalogue }\end{array}$ & & \\
\hline & $V$ & $\begin{array}{c}\text { Author's } C C D \text { magnitudes } \\
\text { catalogue }\end{array}$ & $19^{\prime} \times 24^{\prime}$ & $\sigma_{V}= \pm 0.06 \mathrm{mag}$ \\
\hline \multirow[t]{2}{*}{10} & $B$ & derived from the 2MASS catalogue & & \\
\hline & $V$ & $\begin{array}{c}\text { Author's } C C D \text { magnitudes } \\
\text { catalogue }\end{array}$ & $19^{\prime} \times 24^{\prime}$ & $\sigma_{V}= \pm 0.05 \mathrm{mag}$ \\
\hline
\end{tabular}

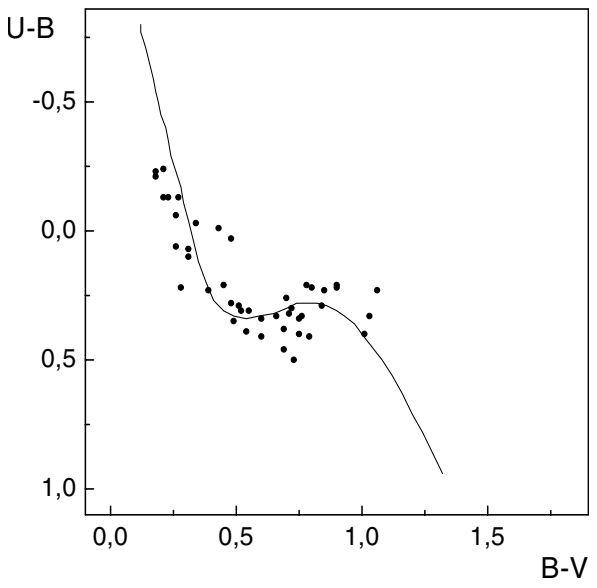

Fig. 5. Two-color diagram of the cluster Trumpler 2.

These possible cluster members are marked by "prob". In Fig. 6 they are plotted by $\triangle$.

The estimation of the age of the cluster is based on the grid of isochrones published by the Padua research group ${ }^{2}$ and described in the paper Girardi et al. (2000). The superposition of various isochrones on the CMD shows the perfect coincidence of the $8.913 \times 10^{7} \mathrm{yr}$ isochrone for $Z=0.019$ with the cluster diagram. The accuracy of determining age depends on the grid step $( \pm 0.05 \log A)$. Corresponding to this isochrone, the turn-off point of the MS is $(B-V)_{0}=-0.16 \mathrm{mag}$.

\footnotetext{
${ }^{2}$ http://www.pleadi.astro.pd.it
}

\begin{tabular}{lcccc}
\hline \hline$N$ & $\begin{array}{c}\text { Radius-vector } \\
(\operatorname{arcmin})\end{array}$ & $\begin{array}{c}M_{V} \\
\mathrm{mag}\end{array}$ & $\begin{array}{c}(B-V)_{0} \\
\mathrm{mag}\end{array}$ & $\begin{array}{c}P \\
\%\end{array}$ \\
\hline $1095^{*}$ & 1.15 & -3.12 & 1.39 & 94 \\
1748 & 17.35 & -2.94 & 1.48 & prob \\
1941 & 36.70 & -3.19 & 0.51 & prob \\
\hline
\end{tabular}

* Suspected variable star.

The cluster luminosity function is plotted for 148 cluster members (Fig. 7a). The cluster mass function was plotted using only stars of the cluster MS located lower than the turn-off point $M_{V}=-0.41 \mathrm{mag}$ (Fig. $7 \mathrm{~b}$ ). Star masses were calculated using the data from the work of Girardi et al. (2000). The straight line drawn by the least-squares-fit with the $95 \%$ confidence level has the slope $\Gamma=-1.90 \pm 0.22$. That is in good agreement with the numerical results of Scalo (1998) in his three-segment power law of the initial mass function for star clusters and associations of the Milky Way and LMC. The Trumpler 2 mass function shows the lack of stars with the mass about $2.8 M_{\odot}$. This feature is also observed on the CMD for the $M_{V} \sim+0.7$.

\section{Variable stars}

In this study of the $80^{\prime} \times 80^{\prime}$ area where star proper motions were obtained, seven variable stars from the the General catalogue (GCVS) of Kholopov (1998) were found. All of them are situated far from the center of the cluster $\left(R>15^{\prime}\right)$ and hardly belong to it, in spite of the the fact that they lie in a $2 \sqrt{2} \sigma$ circle on the VPD. Their data are given in part A of Table 6. Its rows contain 1 - the GCVS star indication; 2 - the authors' number; 3, 4- the right ascention and the declination of the star; 5 - variability type; 6 - the distance from the center; 
Table 6. Variable stars in the region of the open cluster Trumpler 2.

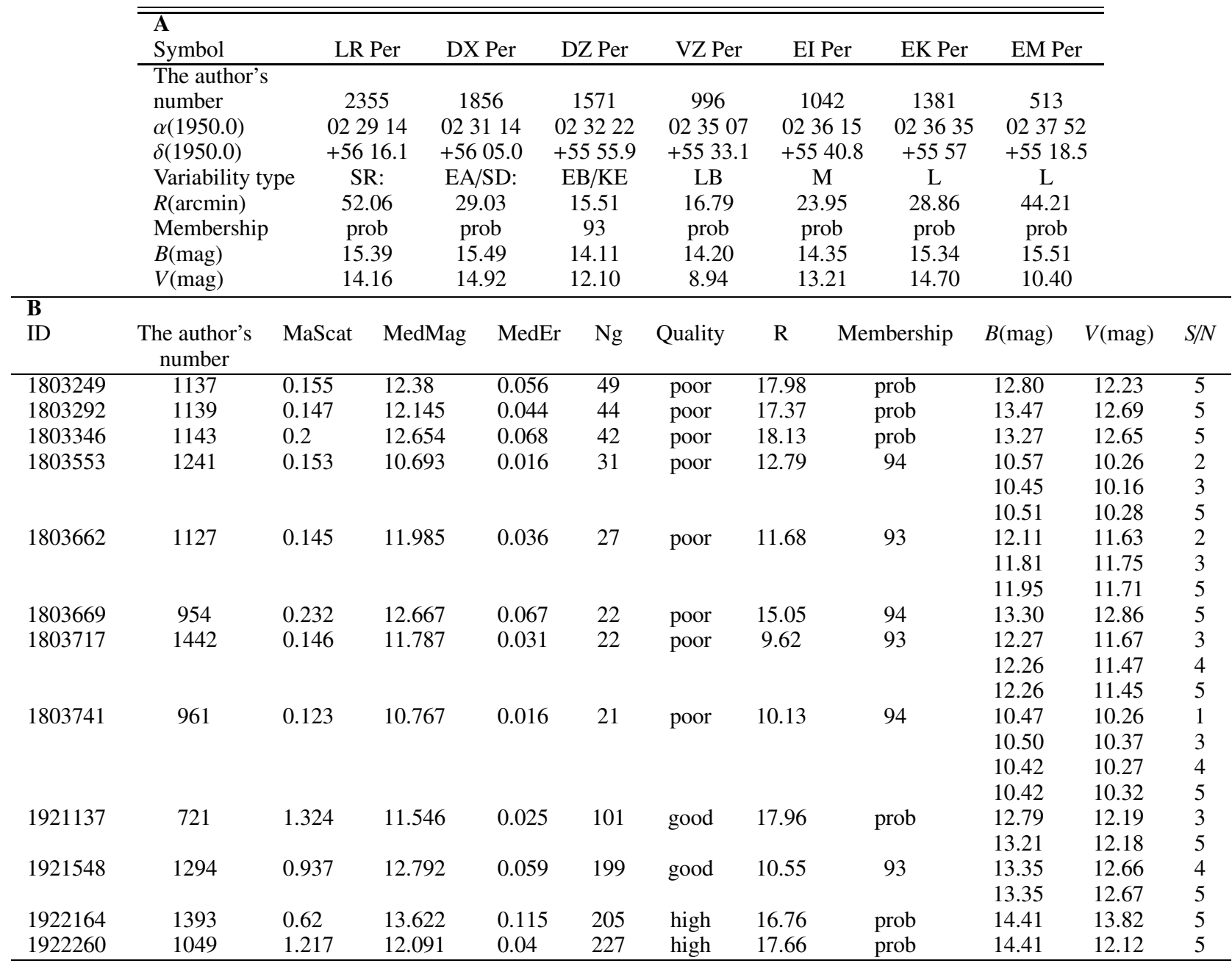

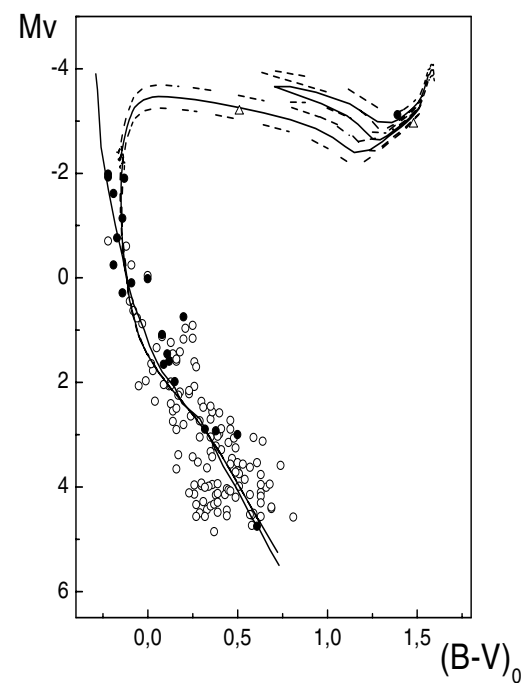

Fig. 6. CMD diagram of all cluster members. ZAMS - Schmidt-Kaler (1965). Isochrones: $z=0.0019$, solid line - age $=8.913 \times 10^{7} \mathrm{yr}$; dot lines - adjacent ones, $\bullet-$ stars supplied by photoelectric photometric data, o - all the other.

7, $8-B, V$ magnitudes from authors photometry catalogue (Table 3); 9 - membership.

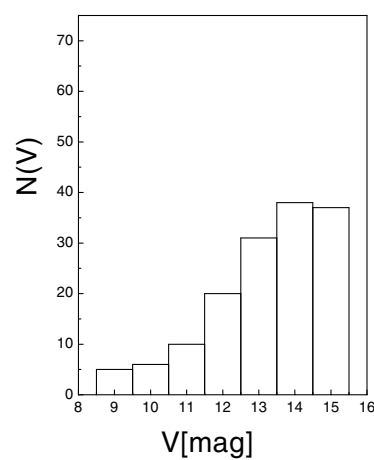

a

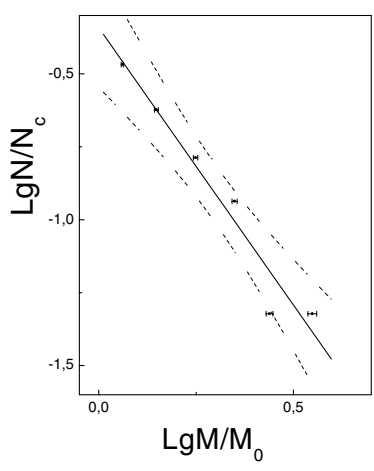

b
Fig. 7. a) Luminosity function and b) mass function of the Trumpler 2.

The search for variable stars was carried out in the recently published database SKYDOT ${ }^{3}$ (SD; Woźniak 2004) relied on the Northen Sky Variability Survey (NSVS). From the list of all the objects presented in the SD we selected only those whose amplitude exceeds more than 3 times the mean error. Twelve of them could be the cluster members. The list of selected objects is given in part B of Table 6. Its columns contain 1 - the SD star indication; 2 - the authors' number; 3 - the maximum

\footnotetext{
${ }^{3}$ http://skydot.lanl.gov
} 
brightness amplitude; 4 - the mean magnitude; 5 - the mean error of the magnitude determination; 6 - the number of the reliable observations during the whole period; 7 - quality of observations; 8 - the distance from the cluster center; 9 - membership; $10,11-B, V$ magnitudes; 12 - the photomety specific number from Table 3. Star magnitudes in the SD were determined in the natural system that was close to the $V$ band. Curves of the brightness variation do not permit determination of the types of the variability because of the low quality of most of the observations. Unfortunately the technical reasons did not permit the investigators to observe the bright stars, which made it impossible to compare their results with the work of the other researchers. This obstacle in particular prevented us from verifying our supposition about the variability of the probable cluster member star N 1045 (the red giant).

Acknowledgements. We would like to thank the investigators of the Pulkovo observatory E. Polyakov and A. Devyatkin for their collaboration in the observations. E. Jilinski thanks the MCT Brazil for financial support.

\section{References}

Bekyashev, R. H., Kanaev, I. I., Devyatkin, A. V., et al. 1998, Izv. Glav. Astron. Obs. Pulkovo, 213, 249, In Russian

Eibighausen, E. C. 1942, AJ, 50, 1

Frolov, V. N., Jilinski, E. G., Ananjevskaja, Yu. K., et al. 2002, A\&A, 396, 125

Girardi, L., Bressan, A., Bertelli, G., \& Chiosi, C. 2000, A\&A, 141, 371

Hoag, A. A., Johnson, H. L., Iriarte, B., et al. 1961, Pub. USNO, 17, 496 Hoag, A. A. 1966, Vistas, 8, 139

Jilinski, E. G., Frolov, V. N., Ananjevskaja, Yu. K., \& Drake, N. A. 2000, Izv. Glav. Astron. Obs. Pulkovo, 215, 57, In Russian

Kadla, Z. I. 1966, Izv. Glav. Astron. Obs. Pulkovo, 181, 93, In Russian

Kholopov, P. N. 1985, General Catalogue of Variable Stars (Moskow: Nauka)

Sanders, W. 1971, A\&A, 14, 226

Sanner, J., Geffert, M., Brunzendorf, J., \& Schmoll 1999, A\&A, 349, 448

Sanner, J., Altmann, M., \& Brunzendorf, J. 2000, A\&A, 357, 471

Sanner, J., Brunzendorf, J., Will, J.-M., \& Geffert, M. 2001, A\&A, 369, 511

Scalo, J. M. 1998, ASP Conf. Ser., 142, 201

Schmidt-Kaler, Th. 1965, Landolt-Bornstein, Neue Series, 298

Skrutskie, et al. 1997, in impact of Large Scale Near-IR Sky Surveys, ed. F. Garzon et al. (Dordrecht: Kluwer), 25

Trümpler, R. J. 1930, Lick Obs. Bull., 14, 154

Wozńiak, P. R., Westrand, W. T., Akerlof, C. V., et al. 2004, Preprint Los Alamos National Laboratory 